\title{
CASTLEMAN'S DISEASE: DIFFERENTIAL DIAGNOSIS OF LYMPHOPROLIFERATIVE DISORDERS IN A PATIENT WITH PRIMARY SJÖGREN SYNDROME
}

Victória Carneiro Dal Moro ${ }^{1, \star}$, Ana Luisa Bagno de Almeida ${ }^{1}$, Samara de Quadros Lobêㄹ ${ }^{1}$ Larissa Maria Oliveira Gonzaga ${ }^{1}$, Fabrícia Fonseca Simil ${ }^{1}$, Maria Raquel da Costa Pinto ${ }^{1}$, Túlio de Paula Vasconcelos ${ }^{1}$, Moisés Salgado Pedrosa ${ }^{1}$, Anna Carolina Faria Moreira Gomes Tavares ${ }^{1}$, Leandro Augusto Tanure ${ }^{1}$

1.Universidade Federal de Minas Gerais, Belo Horizonte (MG), Brazil.

*Corresponding author: vickdalmoro@hotmail.com

\section{BACKGROUND}

Castleman's disease comprises a group of lymphoproliferative disorders that can manifest itself as unicentric form, when only one lymph node chain is involved or as multicentric form, that affects two or more, accompanied by prominent systemic manifestations. Men of the fifth and sixth decades are the most affected and it is strongly associated with autoimmune disease (in addition to HIV infection and gammopathies). Systemic manifestations are mediated by proinflammatory cytokines (IL1, IL2, IL6, VEGF) and they can resemble underlying autoimmune disease.

\section{CASE REPORT}

Male, 49 years old, with primary Sjögren's syndrome (SSp) and silicosis, started intermittent fever, adynamia, loss of appetite, dyspnea, cough, weight loss and foamy urine, in addition to inguinal and axillary lymph node enlargement. Altered laboratory and histopathological findings were anemia and thrombocytopenia with normal bone marrow biopsy, proteinuria secondary to membranous nephropathy (seen on renal biopsy), low complement, hypergammaglobulinemia. Chest and abdominal tomography showed mediastinal lymph node enlargement and pleural effusion. Inguinal lymph node biopsy revealed lymphoid follicles with sclerotic germinal centers penetrated by arterioles and areas of sinus histiocytosis, plasmacytosis and proliferation of postcapillary venules (Fig 1) with negative immunohistochemistry for HHV8 screening. Constitutional symptoms, serositis, cytopenias, proteinuria, more than two compromised lymph node chains, exclusion of other infectious and lymphoproliferative diseases and histopathological findings are compatible with multicentric Castleman's disease.

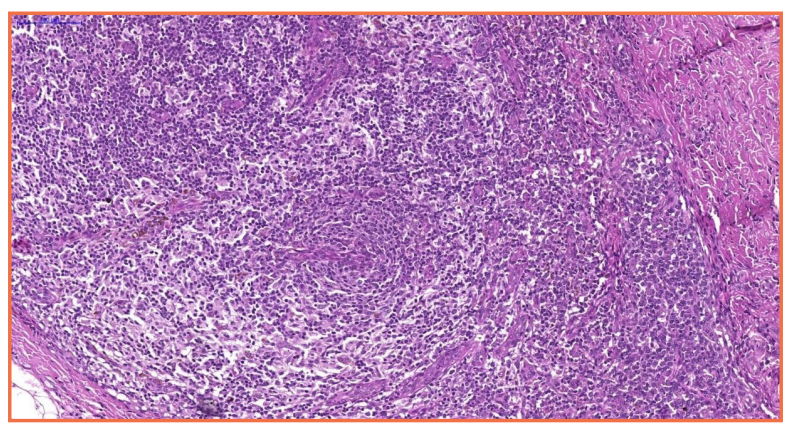

Figure 1. Lymph node representation. There is a prominent germinal center in the middle with a hyalinized arteriole. Lymphocytes are also noted on the periphery of the follicle resulting in an "onion ring" appearance. The interfollicular stroma is prominent with numerous hyperplasic vessels. Hematoxylin and eosin staining.

\section{CONCLUSION}

Lymphoproliferative disorders and Sjögren's syndrome are closely related conditions. They are part of differential diagnosis of lymph node enlargement and constitutional symptoms among patients with autoimmune diseases, in addition to flare of underlying disease and infections. Castleman's disease is rare condition and lymph node biopsy is essential to confirm the disease, with specific findings but not pathognomonic. The literature warns of researching Castleman's disease when the spectrum of an autoimmune disease changes in a context of lymphadenopathy. 